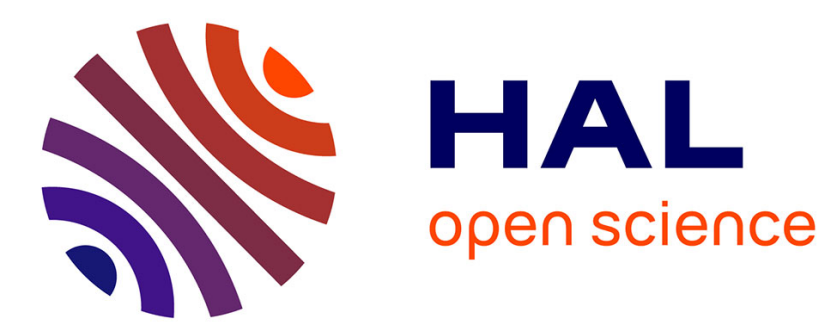

\title{
Control of a Multi-Stage Continuous Fermentor for the study of the wine fermentation
}

Céline Casenave, Denis Dochain, Jérôme Harmand, Marc Perez, Alain

Rapaport, Jean-Marie Sablayrolles

\section{- To cite this version:}

Céline Casenave, Denis Dochain, Jérôme Harmand, Marc Perez, Alain Rapaport, et al.. Control of a Multi-Stage Continuous Fermentor for the study of the wine fermentation. 19th IFAC World Congress 2014, Aug 2014, Cap Town, South Africa. 10.3182/20140824-6-ZA-1003.00627 . hal-01064704

\section{HAL Id: hal-01064704 \\ https://inria.hal.science/hal-01064704}

Submitted on 17 Sep 2014

HAL is a multi-disciplinary open access archive for the deposit and dissemination of scientific research documents, whether they are published or not. The documents may come from teaching and research institutions in France or abroad, or from public or private research centers.
L'archive ouverte pluridisciplinaire HAL, est destinée au dépôt et à la diffusion de documents scientifiques de niveau recherche, publiés ou non, émanant des établissements d'enseignement et de recherche français ou étrangers, des laboratoires publics ou privés. 


\title{
Control of a Multi-Stage Continuous Fermentor for the study of the wine fermentation *
}

\author{
C. Casenave ${ }^{*, * *}$ D. Dochain ${ }^{* * *}$ J. Harmand ${ }^{* * * *, * *}$ M. Perez ${ }^{\dagger}$ \\ A. Rapaport ${ }^{*, * *}$ J-M. Sablayrolles ${ }^{\dagger}$ \\ * INRA, UMR INRA-SupAgro MISTEA, Montpellier 34060, France \\ (e-mail: \{celine.casenave, alain.rapaport\}@supagro.inra.fr) \\ ** INRA-INRIA Project team MODEMIC, Sophia-Antipolis, France. \\ *** CESAME, Université Catholique de Louvain, Louvain-la-neuve \\ 1348,Belgium (e-mail: denis.dochain@uclouvain.be) \\ **** INRA LBE, Narbonne 11100, France \\ (e-mail: Jerome.Harmand@supagro.inra.fr) \\ ${ }^{\dagger}$ INRA UMR SPO, Montpellier 34060, France (e-mail: \\ \{marc.perez,sablayro\}@supagro.inra.fr)
}

\begin{abstract}
Yeasts play a central role in the wine making process. To study the yeasts in a stable environment and physiological state, a Multi-Stage Continuous Fermentor (MSCF) has been designed that mimics steps of the batch fermentation process. In this paper, the problem of the control of the sugar concentrations in each of the four reactors of the MSCF is considered. The cascade structure of the device leads to a constraint on the input flow rates (the control variables). A control strategy based on a linearizing control law coupled with a state observer and an anti windup component is proposed and finally implemented on the experimental process.
\end{abstract}

Keywords: wine fermentation; cascade of reactors; control design; varying input constraints.

\section{INTRODUCTION}

The alcoholic fermentation is a fundamental step of the wine-making process. During the fermentation, which is performed in a batch reactor, the sugar contained in the grape juice is converted in ethanol and other compounds which give the wine all its aroma. The control of the fermentation process is therefore of importance; in particular, it requires a good knowledge of the yeasts and their physiology. However, studying the yeasts during the batch fermentation process is not easy because the physiological state of the yeasts change all along the fermentation: in particular, the yeasts go through a growth phase and a stationary phase. In Clement et al. [2011] the use of a Multi-Stage Continuous Fermentor (MSCF) has been proposed to mimic the batch wine fermentation process. It is composed of 4 reactors connected in series; at each stage, the input of the reactor corresponds to a fraction of the output of the preceding reactor. With such a device, the yeasts obtained at equilibrium in each stage are in a stable physiological state which corresponds to a key transient state of the batch fermentation. Indeed, the MSCF enables to pass from a time scale (the transient states of the batch fermentation) to a space scale (the equilibrium states of the 4 reactors of the MCSF). Thus, it is possible to get simultaneously (in the 4 reactors) some yeasts in the growth phase and others in the stationary

\footnotetext{
$\star$ The work is supported by the 7th Framework Program of the European Union (CAFE Project - Large Collaborative Project KBBE-2007-2-3-01).
}

phase, in a (time) stable environment and physiological state. Some measurements and experimental tests can then be performed on the yeasts; compared to results obtained from batch fermentations, the obtained results are here completely independent of the reaction kinetics.

The equilibrium points reached in each of the 4 reactors of the MSCF can be characterized in term of the sugar concentration. The problem considered in this paper is the control of the sugar concentration of the 4 reactors. The values of the sugar concentration at equilibrium depends on the value of the input flow rates of the reactors, which are therefore considered as the control inputs.

The control of a continuous bioreactor has been widely studied in automatic control literature [Bastin and Dochain 1990]. This problem is currently encountered in practice, in particular for bioengineering processes used, for example, for the waste water treatment [Katebi et al. 1999, Olsson and Newell 1999, Dochain and Vanrolleghem 2001]. In comparison, only a few papers deal with the problem of the control of a cascade of bioreactors [Vigie et al. 1990, Simeonov et al. 2011], whereas such kind of devices is often used in industrial engineering processes [Chen 1992, Hill and Robinson 1989, de Gooijer et al. 1996, Harmand et al. 1999]. Moreover, the control problems on multistage continuous reactors which have already been studied deal most of the time with the control of a unique quantity. In [Vigie et al. 1990] for example, the authors indeed design a controller for the glucose concentration in the process outlet. In [Simeonov et al. 2011], the objective is 
the maximization of the biogas production in a two-stage anaerobic bioreactor. However, the authors only propose a strategy that maximizes the biogas flow rate of the first reactor, the second one being not controlled.

In this paper, the goal is to control the sugar concentration in each stage of the reactor with, as control inputs, the four input flow rates. Because of the cascade structure of the process, the values of the control inputs are constrained: indeed, the input flow rate of a stage is necessarily smaller than the output flow rate of the preceding stage. In this paper, a control strategy is proposed, based on a linearizing control law coupled with a state observer and an anti-windup loop. The closed loop system stabilizes faster than when a constant input is applied ${ }^{1}$. It has been validated both in numerical simulation, and on the experimental process.

The paper is organized as follows. In Section 2 the experimental set up is described. The model used for the design of the control law is presented and validated in Section 3. Section 4 deals with the design of the control strategy. Finally in section 5 , the results obtained on the experimental process are presented.

\section{PROCESS DESCRIPTION}

The experimental set up has been developed by the research unit SPO (Sciences for Oenology) of INRA (Montpellier). It is composed of 4 reactors connected in series (see Figure 1). The output flow rate of each reactor is equal to its input flow rate, in such way that the volumes $V_{i}, i=1: 4$ of the reactors remain constant (as in classical chemostats). The first reactor is fed with a synthetic medium which simulates a grape juice. The other reactors are fed by the outlet medium of the preceding reactor. The input flow rate $Q_{i}, i=1: 4$ of the reactors are controlled independently by some piston pumps, the only constraint (coming from the cascade structure of the device) being that the input flow rate $Q_{i}$ of the $i^{\text {th }}$ reactor has to be lower than the output flow rate $Q_{i-1}$ of the $(i-1)^{\text {th }}$ reactor:

$$
0 \leq Q_{4} \leq Q_{3} \leq Q_{2} \leq Q_{1} \leq Q_{\max }
$$

with $Q_{\max }$ the maximal flow rate which can be applied (i.e. each tank is fed from the input of the previous one without any external addition, excepted for the first tank). The temperature of the medium in each reactor is regulated at $28^{\circ} \mathrm{C}$. Only the $\mathrm{CO}_{2}$ production rate (in each of the 4 reactors) is measured on line. A scheme of the MSCF is given in Figure 1.

\section{MODEL DESCRIPTION AND VALIDATION}

\subsection{Model of the batch fermentation}

The model of the batch fermentation considered in this paper is the one described in David et al. [2010]. It is based on the following reaction scheme:

- the yeasts $X$ grow on the nitrogen $N$, with a yield coefficient $k_{1}$ :

$$
N \longrightarrow k_{1} X
$$

1 The minimal time synthesis presents bang-bang and singular arcs (see Bayen et al. [2013]). It is consequently sensitive to error measurements and therefore difficult to implement in practice

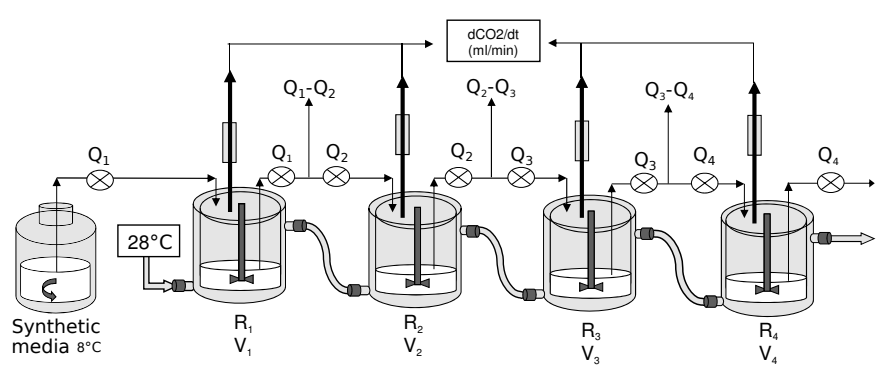

Fig. 1. Scheme of the Multi Stage Continuous Fermenter (MSCF).

- the sugar $S$ is enzymatically degraded in ethanol $E$ and $\mathrm{CO}_{2}$, and inhibited by the ethanol, with a yield coefficient $k_{2}$ :

$$
S \stackrel{X}{\longrightarrow} k_{2} E+k_{2} \mathrm{CO}_{2}
$$

The mass balance model is written as follows :

$$
\begin{cases}\frac{d X}{d t}=\mu_{1}(N) X ; & \frac{d N}{d t}=-k_{1} \frac{d X}{d t} \\ \frac{d E}{d t}=\mu_{2}(E, S) X ; & \frac{d S}{d t}=-k_{2} \frac{d E}{d t}\end{cases}
$$

with:

$$
\mu_{1}(N)=\frac{\mu_{1}^{\max } N}{K_{N}+N}, \quad \mu_{2}(E, S)=\frac{\mu_{2}^{\max } S}{K_{S}+S} \frac{K_{E}}{K_{E}+E},
$$

where $X, N, E$ and $S$ are the concentrations of the yeast, the nitrogen, the ethanol and the sugar (in $g . L^{-1}$ ) respectively; and $k_{1}, k_{2}, \mu_{1}^{\max }, \mu_{2}^{\max }, K_{E}$ and $K_{S}$ are some positive constants. To complete the model, the following initial conditions are considered:

$$
X(0)=X^{i n}, N(0)=N^{i n}, E(0)=0, S(0)=S^{i n} .
$$

The $C_{2}$ production rate is indeed here equal to $\mu_{2}(E, S) X$

\subsection{Model of the MSCF}

The model of the MSCF is obtained from the model of the batch fermentation by addition of terms related to the flow rates: $\forall i=1: 4$,

$$
(\mathcal{P})\left\{\begin{array}{rrrr}
\frac{d X_{i}}{d t}= & & \mu_{1}\left(N_{i}\right) X_{i}+D_{i}\left(X_{i-1}-X_{i}\right) \\
\frac{d N_{i}}{d t}= & & -k_{1} \mu_{1}\left(N_{i}\right) X_{i}+D_{i}\left(N_{i-1}-N_{i}\right) \\
\frac{d E_{i}}{d t}= & \mu_{2}\left(E_{i}, S_{i}\right) X_{i}+D_{i}\left(E_{i-1}-E_{i}\right) \\
\frac{d S_{i}}{d t}= & -k_{2} \mu_{2}\left(E_{i}, S_{i}\right) X_{i}+D_{i}\left(S_{i-1}-S_{i}\right)
\end{array}\right.
$$

where $D_{i}=\frac{Q_{i}}{V_{i}}$ is the dilution rate of the $i^{\text {th }}$ reactor, $\left(X_{0}, N_{0}, E_{0}, S_{0}\right)=\left(0, N^{i n}, 0, S^{i n}\right)$ with $N^{i n}, S^{i n}>0$, and $\mu_{1}, \mu_{2}, k_{1}, k_{2}, \mu_{1}^{\max }, \mu_{2}^{\max }, K_{E}$ and $K_{S}$ are the same functions and parameters than the ones of the batch fermentation model [Clement et al. 2011]. In the sequel, we denote $C_{i}$ the $\mathrm{CO}_{2}$ production rate of the $i^{\text {th }}$ reactor:

$$
C_{i}:=\mu_{2}\left(E_{i}, S_{i}\right) X_{i}
$$

\subsection{Comparison between batch fermentation and multistage continuous fermentation}

The design of the experimental is indeed based on the notion of equivalence between batch reactors and plug flow reactors in steady state, and the related notion of space time, widely used in reactor design (e.g. Levenspiel [1999]). 
If the input medium of the plug flow reactor is the same than the initial medium of the batch fermentation, then the equilibrium state reached inside the plug flow reactor has the same space evolution than the time evolution of the state variable during the batch fermentation.

The cascade of reactors is indeed aimed at approximating the ideal plug flow reactor behavior (see also e.g. Levenspiel [1999]). In Clement et al. [2011], the concentrations of yeast and nitrogen, and the $\mathrm{CO}_{2}$ production rate obtained at equilibrium in the 4 reactors of the MSCF for different values of the dilution rates $D_{i}$ are compared to the time evolution of the same quantities obtained with batch fermentation. The obtained results make the authors conclude that the MSCF enables to mimic, at equilibrium, the transient states of the batch fermentation.

From a modeling viewpoint, the MSCF corresponds to a finite difference approximation of the plug flow reactor. This discretization necessarily induces errors; the quality of the approximation will in particular depend on the number of reactors considered. Many numerical studies have shown that in practice a small number of tanks in series, typically 3 , with possibly different volumes, is sufficient to well approximate the behavior of a plug flow reactor [Hill and Robinson 1989]. In Clement et al. [2011], they consider that 4 reactors are enough to obtain a good approximation (according to their needs).

There is indeed another source of errors in the approximation : the input medium of the MSCF is not exactly the same than the initial medium of the batch fermentation. They have the same nitrogen and sugar concentrations, but the yeast concentrations are not equal. In fact there is no yeast in the inlet of the MSCF: the yeasts are inoculated in each reactor of the MSCF, at the starting of the process, as for the batch fermentation. Fortunately this difference does not decrease the quality of the approximation. This is due to the small value of the initial yeast concentration (around $10^{6} \mathrm{cell} / \mathrm{ml}$ ), and to the fact that the values of the concentrations are compared in terms of functions of the fermentation progress $1-\frac{S(t)}{S^{i n}}$ instead of the time.

The same comparison as the one made in Clement et al. [2011] with experimental data has been performed with numerical simulations. In Figure 2, the yeast concentration $X$ and the $C_{2}$ production rate $\mu_{2}(E, S) X$ are plotted versus the fermentation progress $1-\frac{S}{S^{i n}}$. The plotted values correspond for one part to the transient states of a batch fermentation and for the other part to the equilibrium states reached in the 4 reactors of the MSCF for dilution rates $D_{i} \in\{0.05,0.1,0.15,0.2,0.25\} \forall i=1: 4$, such that ${ }^{2} D_{1} \geqslant D_{2} \geqslant D_{3} \geqslant D_{4}$. The results are similar to the ones obtained on the experimental process.

\subsection{Model parameter identification and comparison with experimental data}

The values of the parameters of model (7), except the one of ${ }^{3} k_{2}$, have been identified from experimental data: a simplex method was used to minimize the sum of the squares of the distances between the experimental

\footnotetext{
2 We consider here 4 reactors with the same volume $V:=V_{i}, i=1: 4$.

3 The value of parameter $k_{2}$ is considered as known; we used the value given in David et al. [2010].
}
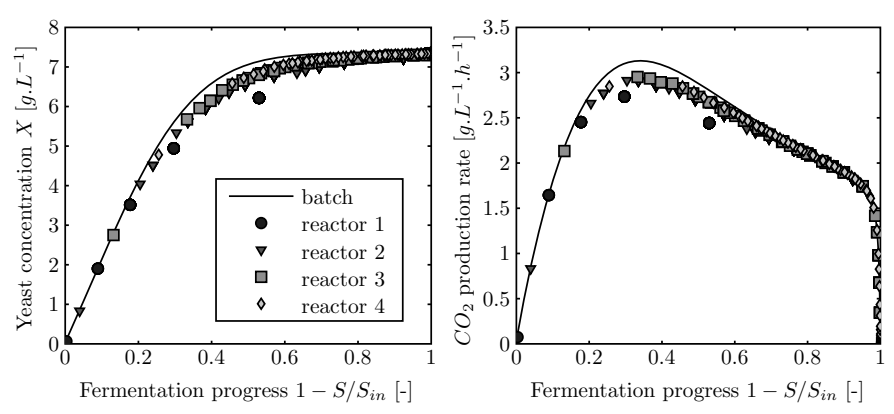

Fig. 2. Numerical comparison between batch fermentation and equilibrium states of the MSCF.

measurements of the sugar concentration at equilibrium in the 4 stages of the MSCF and the values obtained by numerical simulation of the model. We finally get :

$\begin{array}{lll}k_{n}=1.57\left[g . L^{-1}\right], & k_{1}=0.0606[-], & \mu_{1}^{\max }=1.34\left[h^{-1}\right], \\ k_{s}=0.0154\left[\mathrm{~g} \cdot \mathrm{L}^{-1}\right], & k_{2}=2.17[-], & \mu_{2}^{\max }=1.45\left[h^{-1}\right], \\ k_{e}=14.1\left[\mathrm{~g} \cdot \mathrm{L}^{-1}\right] . & & \end{array}$

The comparison between the experimental data used for the identification process, and the values obtained by simulation with the above set of parameter values is given in Figure 3 (top). For the cross validation, we also compared simulated trajectories of a batch fermentation with experimental data: the results are given in Figure 3 (bottom).
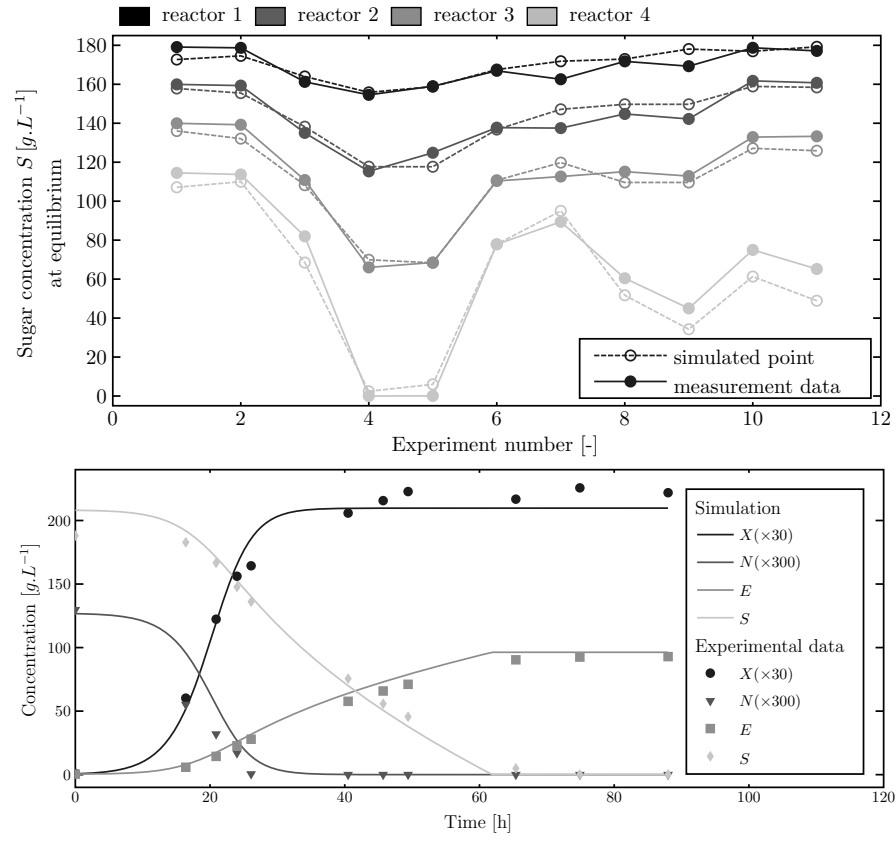

Fig. 3. Comparison between experimental data and simulations of the MSCF model. Top: sugar concentration values at equilibrium. The experimental data are those have been used for the identification process. Bottom: yeast, nitrogen, ethanol and sugar concentrations of a batch fermentation (cross validation).

\section{DESIGN OF THE CONTROL LAW}

First recall that the objective is to control the sugar concentration in each stage of the MSCF, the control inputs being the input flow rates $Q_{i}, i=1: 4$, of the 4 reactors. In the sequel, we denote $S_{i}^{*}$ the value of the 
sugar concentration setpoint in the $i^{\text {th }}$ reactor (for $i=$ $1: 4)$. We only consider setpoint values such that there exist constant input control values $\left(Q_{1}^{*}, Q_{2}^{*}, Q_{3}^{*}, Q_{4}^{*}\right) \in$ $\left[0, Q_{\max }\right]^{4}$ verifying the constraint (1) and which, when applied to system (7), stabilize the sugar concentration in the 4 reactors at the values $S_{i}^{*}, i=1: 4$. Note that this implies that $S^{i n}>S_{1}^{*}>S_{2}^{*}>S_{3}^{*}>S_{4}^{*}>0$.

The control design is facing two main difficulties: on one hand, no on-line measurement of the sugar concentration is available, and on the other hand the control inputs $Q_{i}$ have to verify the constraint (1). The proposed control strategy is based on a linearizing control law, coupled with a state observer and an anti-windup component. A scheme of the control strategy is given in Figure 4.

In order to account for the system nonlinearities, a linearizing control law is first considered. The equation of $S_{i}$ has a relative degree equal to 1 (with respect to the control input $\left.Q_{i}\right)$; the control law is therefore written as follows :

$$
\widetilde{Q}_{i}=V_{i} \frac{k_{2} C_{i}+v_{i}}{S_{i-1}-S_{i}}:=\Psi_{i}\left(v_{i}, S, C\right),
$$

where $C=\left(C_{1}, C_{2}, C_{3}, C_{4}\right)^{T}, S=\left(S_{1}, S_{2}, S_{3}, S_{4}\right)^{T}$ and $v_{i}$ is the expression of the desired closed loop dynamic of $S_{i}$. Here we consider a simple proportional-integral linear dynamics:

$$
v_{i}=a_{i, 1}\left(S_{i}^{*}-S_{i}\right)+a_{i, 2} \int_{0}^{t}\left(S_{i}^{*}(\tau)-S_{i}(\tau)\right) d \tau,
$$

with $a_{i, 1}$ and $a_{i, 2}$ some positive constants. The integral term is introduced to compensate modeling and measurements errors.

The linearizing control law (9) assumes a full knowledge of both the sugar concentrations $S_{i}, S_{i-1}$ and the $\mathrm{CO}_{2}$ production rate $C_{i}$. However, only the $\mathrm{CO}_{2}$ production rate is measured on-line. To get an on-line estimate of the sugar concentration, we use the following asymptotic observer [Bastin and Dochain 1990] : $\forall i=1: 4$,

$$
(\mathcal{O})\left\{\begin{aligned}
\frac{d \hat{S}_{i}}{d t}(t) & =-k_{2} C_{i}^{m}(t)+D_{i}(t)\left(\hat{S}_{i-1}(t)-\hat{S}_{i}(t)\right) \\
\hat{S}_{i}(0) & =\hat{S}_{i}^{0},
\end{aligned}\right.
$$

with $\hat{S}_{0}(t)=S^{i n}, \forall t>0$ and $C_{i}^{m}(t)$ the measurement of $C_{i}(t)$. The rate of convergence of such an observer can not be adjusted: it depends on the value of $D_{i}$. However, several other observation strategies (high gain observer, extended Kalman Filter, etc) have been tested, and no ones give better results: the convergence rate is always limited by the value of $D_{i}$. We therefore have chosen to consider the asymptotic observer $(\mathcal{O})$ which has the advantage to be very simple and robust because it requires the only knowledge of the parameter $k_{2}$ of the model, the value of which is reliable. Note that the observation problem with production rate measurement in the chemostat is difficult because of the existence of "bad" inputs. When an input $D_{i}(\cdot)$ is set to zero, the estimator does not converge asymptotically. One may then possibly switch to another observer specifically designed for the zero input (see [Rapaport and Maloum 2004] for a similar situation), but one can check that even in this simpler case, it is not possible to write the system in the observability form [Gauthier and Kupka 2001]. Theoretically, there should exists an observer of Kazantsis-Kravaris form (or an approximation) [Andrieu and Praly 2006] but it remains an open problem to write it explicitly.

Finally, the quality of the estimation of $S$ will essentially depend on the quality of the initialization of the observer. In practice, the control law will be used to go from one equilibrium point to another. As a consequence, we assume that, at the observer initialization time $t=0$, the system is at equilibrium. Under this assumption, we get, from (7), the following estimation $\hat{S}_{i}^{0}$ of $S_{i}(0)$ :

$$
\hat{S}_{i}^{0}=-k_{2} \frac{C_{i, m}^{0}}{D_{i}(0)}+\hat{S}_{i-1}^{0}, i=1: 4 .
$$

where $C_{i, m}^{0}$ is the measurement of $C_{i}(0)$ and $\hat{S}_{0}^{0}=S^{i n}$. In practice, some off-line measurements of the sugar concentration are available. The information given by these measurements, even if they are available only a few hours after the sampling, will be used to adjust the estimation of the observer (see section 5).

The values $\widetilde{Q}_{i}$ of $Q_{i}$ given by the control law (9) do not necessarily meet the constraint (1). To take this constraint into account, we use the following saturation operator :

$$
\operatorname{sat}\left(u ; u_{m}, u_{M}\right):= \begin{cases}u_{M} & \text { if } u \geqslant u_{M} \\ u & \text { if } u_{m}<u<u_{M} \\ u_{m} & \text { if } u \leqslant u_{m} .\end{cases}
$$

Compared to problems often considered in the literature, the constraints on the control inputs are coupled and time-varying. We chose to apply the saturation operator (12) to one control input after the other. The choice of the saturation order of the 4 control inputs $Q_{i}$ is not obvious: it can lead to very different control performances, depending on the setpoints, the initial conditions, and the experimental conditions. The 14 possible saturation orders are given in Table $1, n_{i}=j$ meaning that $Q_{i}$ will be the $j^{\text {th }}$ control input to be saturated. For example, the order

\begin{tabular}{ccccccccccccccc} 
& 1 & 2 & 3 & 4 & 5 & 6 & 7 & 8 & 9 & 10 & 11 & 12 & 13 & 14 \\
\hline$n_{1}$ & 1 & 1 & 1 & 1 & 1 & 2 & 2 & 2 & 3 & 2 & 2 & 3 & 4 & 3 \\
$n_{2}$ & 2 & 2 & 3 & 3 & 4 & 1 & 1 & 3 & 2 & 3 & 4 & 2 & 3 & 4 \\
$n_{3}$ & 3 & 4 & 2 & 4 & 3 & 2 & 3 & 1 & 1 & 4 & 3 & 3 & 2 & 2 \\
$n_{4}$ & 4 & 3 & 3 & 2 & 2 & 3 & 2 & 2 & 2 & 1 & 1 & 1 & 1 & 1 \\
\hline \multicolumn{1}{c}{ Table 1. The 14 possible saturation orders. }
\end{tabular}

$n^{\circ} 8$ (which is the one used for experiments presented in Section 2) has to be understood as follows:

$$
\begin{aligned}
& \text { 1. } n_{3}=1 \\
& \text { 2. } n_{1}=n_{4}=2 \Longrightarrow\left\{\begin{array}{l}
Q_{3}=\operatorname{sat}\left(\widetilde{Q}_{3} ; 0, Q_{\max }\right) \\
Q_{1}=\operatorname{sat}\left(\widetilde{Q}_{1} ; Q_{3}, Q_{\max }\right) \\
Q_{4}=\operatorname{sat}\left(\widetilde{Q}_{4} ; 0, Q_{3}\right) \\
Q_{2}=3=\operatorname{sat}\left(\widetilde{Q}_{2} ; Q_{3}, Q_{1}\right)
\end{array}\right.
\end{aligned}
$$

The saturation strategy can be expressed as a recurrence of the form:

$$
Q_{i}=\operatorname{sat}\left(\widetilde{Q}_{i} ; \widetilde{Q}_{i, m}\left(Q_{j, n_{j}<n_{i}}\right), \widetilde{Q}_{i, M}\left(Q_{j, n_{j}<n_{i}}\right)\right)
$$

where $\widetilde{Q}_{i, m}$ and $\widetilde{Q}_{i, M}$ depend on the chosen order.

It is well known that enforcing input control constraints by application of a saturation operator can result in poor closed-loop performance and overshooting of the integral term. To minimize the performance loss, some anti-windup control schemes have been developed. However, the problems studied in the literature are most of the time linear. In Doyle III [1999] and Kendi and Doyle III [1997], the 
authors propose some anti-windup schemes for SISO and MIMO control affine nonlinear systems with constrained inputs. The anti-windup scheme we considered here comes from these works. It is based on one hand on the transformation of the constraint on $Q_{i}$ in a constraint on $v_{i}$. Indeed, as the function $\Psi_{i}$ defined in (9) is linear with respect to $v_{i}$, we have:

$$
\begin{gathered}
Q_{i+1}<Q_{i}<Q_{i-1} \\
\Leftrightarrow \Phi_{i, i+1}\left(v_{i+1}, S, C\right)<v_{i}<\Phi_{i, i-1}\left(v_{i-1}, S, C\right),
\end{gathered}
$$

where $\Phi_{i, j}$ depends on $\Psi_{i}$ and $\Psi_{j}$. In our case, we have:

$$
\Phi_{i, j}\left(v_{j}, S, C\right)=\frac{S_{i-1}-S_{i}}{V_{i}} \Psi_{j}\left(v_{j}, S, C\right)-k_{2} C_{i} .
$$

Consider some values $\left(\tilde{v}_{1}, \tilde{v}_{2}, \tilde{v}_{3}, \tilde{v}_{4}\right)$ which do not verify the transformed constraint (13). By applying the same saturation order than for the $Q_{i}, i=1: 4$ values, we can express the transformed saturation strategy as follows :

$$
v_{i}=\operatorname{sat}\left(\tilde{v}_{i} ; \tilde{v}_{i, m}\left(v_{j, n_{j}<n_{i}}, C, S\right), \tilde{v}_{i, M}\left(v_{j, n_{j}<n_{i}}, C, S\right)\right) \text {, }
$$
where $\tilde{v}_{i, m}$ and $\tilde{v}_{i, M}$ depend on the chosen order.

The values of $\tilde{v}_{i}$ are then given by (see Figure 4$)$ :

$$
\mathcal{L}\left(\tilde{v}_{i}\right)(p)=K_{1}(p) \mathcal{L}\left(S_{i}^{*}-\hat{S}_{i}\right)(p)-K_{2}(p) \mathcal{L}\left(v_{i}\right)(p)
$$

where $\mathcal{L}$ stands for the Laplace transform and with

$$
K_{1}(p)=a_{i, 1} \text { and } K_{2}(p)=-\frac{a_{i, 2}}{a_{i, 1} p+a_{i, 2}},
$$

$a_{i, 1}$ and $a_{i, 2}$ being chosen in such way that the control law obtained with $v_{i}$ given by (10) without saturation constraint gives good performances.

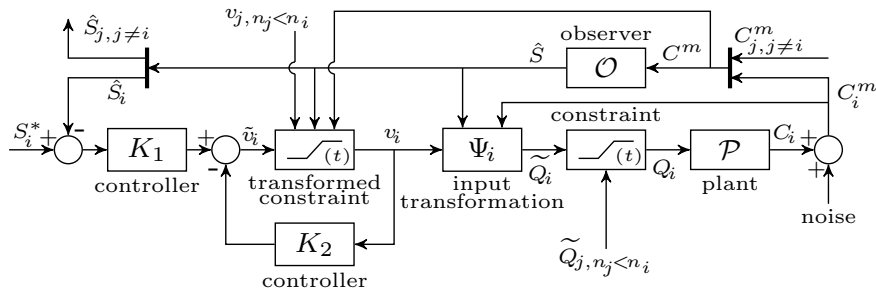

Fig. 4. Control strategy diagram of the $i^{\text {th }}$ reactor.

\section{EXPERIMENTAL VALIDATION}

The proposed control strategy has been validated first on numerical simulations, then on the experimental set up.

The synthetic media used for the experiments contained $S_{i n}=200 \mathrm{~g} \cdot \mathrm{L}^{-1}$ of glucose, and $N_{i n}=0.465 \mathrm{~g} \cdot \mathrm{L}^{-1}$ of assimilable nitrogen. At the beginning of the experiment, the yeasts were inoculated in each reactor, with an initial concentration $X_{\text {in }}$ around $10^{6} \mathrm{cell} / \mathrm{ml}$ which corresponds to $0.04 g . L^{-1}$ for the yeast strain used (the commercial strain EC1118, Lallemand SA).

The values of the setpoints $S_{i}^{*}$, the volumes $V_{i}$ and the initial dilution rates $D_{i}(0)$ are given in Table 2 .

For the control law, the following parameters values were considered: $k_{2}=2.17[-], Q_{\max }=0.25\left[L . h^{-1}\right]$, and $\forall i=$ $1: 4, a_{i, 1}=1.2\left[h^{-1}\right], a_{i, 2}=1\left[h^{-2}\right]$.

For technical reasons (not detailed here), the constraint

\begin{tabular}{|c|c|c|c|c|c|c|}
\hline \multirow{2}{*}{$\begin{array}{c}\text { reactor } \\
\text { number } \\
i\end{array}$} & \multicolumn{4}{|c|}{$\begin{array}{c}\text { Sugar concentration } \\
\left.\qquad g . L^{-1}\right]\end{array}$} & \multirow{2}{*}{$\begin{array}{c}\text { Volume } \\
{[L]} \\
V_{i}\end{array}$} & \multirow{2}{*}{$\begin{array}{c}\text { Initial dilution } \\
\text { rate }\left[h^{-1}\right] \\
D_{i}(0)\end{array}$} \\
\hline & $S_{i}^{*}$ & $S_{i}$ & $S_{i}^{*}-S_{i-1}^{*}$ & $S_{i}-S_{i-1}$ & & \\
\hline 1 & 184 & 177.9 & 16 & 22.1 & 1 & 0.17 \\
\hline 2 & 160 & 152.9 & 24 & 25.0 & 0.7 & 0.19 \\
\hline 3 & 140 & 131.2 & 20 & 21.7 & 0.5 & 0.18 \\
\hline 4 & 50 & 43.3 & 90 & 87.9 & 0.7 & 0.12 \\
\hline
\end{tabular}

(1) has been replaced by the stronger following one:

$$
Q_{1}<Q_{\max } \text { and } \forall i=2: 4,0<Q_{i}<0.9 Q_{i-1} .
$$

The saturation strategy presented in section 4 has therefore been modified consequently.
Table 2. Sugar concentration setpoints and final measurements, volumes and initial dilution rates of the experiment. $S_{0}=S_{0}^{*}=200 \mathrm{~g} \cdot \mathrm{L}^{-1}$

The experimental results are presented in Figure 5. The estimated sugar concentrations given by the observer are first plotted in Figure 5.a. In Figures 5.b and 5.c are given the on-line measurement of the $\mathrm{CO}_{2}$ production rates $C_{i}$ and the computed control input values $D_{i}$. During the
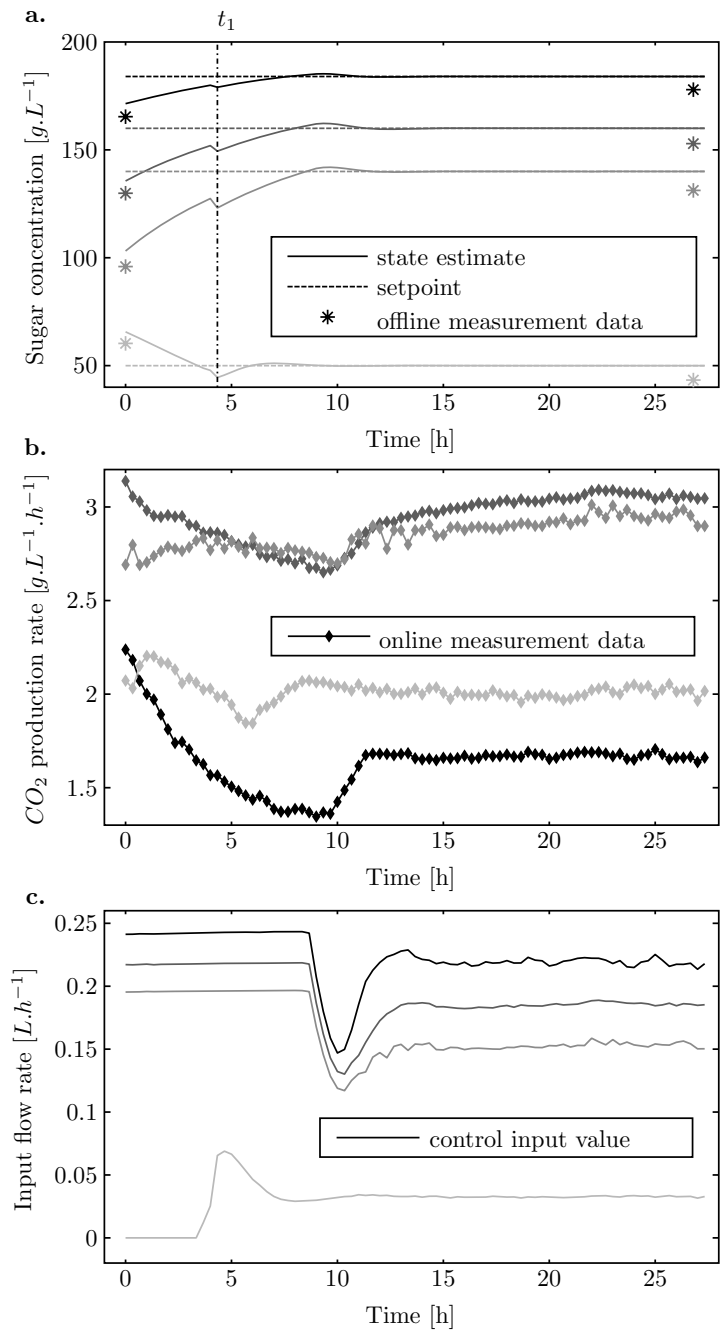

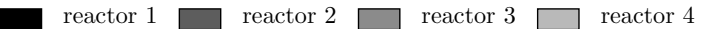

Fig. 5. Experimental results of the control of the sugar concentrations in the 4 reactors of the MSCF.

experiment, two off-line measurements of the sugar concentration were performed: one at the beginning of the experiment, and the other one at the end. The measurements of the initial sugar concentrations were available only at time $t_{1}=4.33 h$, and have been taken into account in the control strategy at this time, to adjust the estimation of the observer. The effects of such an adjustment are visible in Figure 5.a. The measurements of the final sugar 
concentrations are given in Table 2 for comparison with the setpoint values.

As we can see, the qualitative behavior of the control law is good and similar to the one obtained in numerical simulation. However, without any on-line sugar concentration measurement, it is not possible to completely cancel the control error, which is even though smaller than $8.8 \mathrm{~g} . \mathrm{L}^{-1}$ in all the reactors.

The control strategy proposed in this paper does not really control the sugar concentration in each reactor, but rather the differences of sugar concentration between 2 consecutive reactors. The comparison between the values of the difference between consecutive setpoints and measurements (see Table 2) shows that the position error essentially comes from the first reactor. Indeed, for $i=2: 4$, the absolute value of the difference between $S_{i}-S_{i-1}$ and $S_{i}^{*}-S_{i-1}^{*}$ is smaller than $2.1 \mathrm{~g} \cdot \mathrm{L}^{-1}$, whereas it is equal to $6.1 \mathrm{~g} \cdot \mathrm{L}^{-1}$ for $i=1$. Two main reasons can be given to explain the huge value of the error obtained in the first reactor. Firstly, this error depends on the value of $S_{i n}$ which can be ill-known. And secondly, the first reactor of the MSCF corresponds to the starting of the batch fermentation which is a complex and the most ill-known stage of the fermentation. During this phase, a part of the $\mathrm{CO}_{2}$ produced is dissolved in the medium and can therefore not be measured.

\section{CONCLUSION}

In this paper, the problem of the control of the sugar concentration in each of the reactors of a Multi-Stage Continuous Fermenter (MSCF) is studied. Because of the structure of the system (cascade), the control inputs (that is the input flow rates of each reactor) are constrained: the input flow rate of a reactor is necessarily smaller than the one of the preceding reactor. To solve this problem, a control strategy has been proposed: it is based on a linearizing control law coupled with an observer and an antiwindup component. The control law has been validated first on numerical simulation and then on experiments. The obtained results are convincing. However, we still have a position error due to the lack of on-line sugar concentration measurement. This error essentially comes from the first reactor of the MSCF. To mitigate this problem, a solution would consist in controlling the nitrogen or yeast concentration instead of the sugar one in the first reactor. Indeed, in the first reactor, the equilibrium state can indifferently be characterized by the nitrogen, the yeast or the sugar concentration, which is not the case of the other reactors, in which the nitrogen concentration can be too small and the yeast concentration at its maximum value.

\section{ACKNOWLEDGEMENTS}

Special thanks to our former PhD student J. Fernandez, enrolled in the European Project CAFE, but that unfortunately could not have carried on his first investigations.

\section{REFERENCES}

Andrieu, V. and Praly, L. (2006). On the Existence of a Kazantzis-Kravaris/Luenberger Observer. SIAM Journal of Control and Optimization, 45(2), 432-456.
Bastin, G. and Dochain, D. (1990). On-line estimation and adaptive control of bioreactors (Process measurement and control,1). Elsevier, Amsterdam.

Bayen, T., Rapaport, A., and Sebbah, M. (2013). Optimal synthesis for a minimum time problem in the plane with a triangle control set.

Chen, L. (1992). Modelling, identifiability and control of complex biotechnological systems. Ph.D. thesis, Center for Systems Engineering and Applied Mechanics, Universit Catholique de Louvain-la-neuve, Belgium.

Clement, T., Perez, M., Mouret, J., Sablayrolles, J., and Camarasa, C. (2011). Use of a continuous multistage bioreactor to mimic winemaking fermentation. International journal of food microbiology, 150(1), 42-49.

David, R., Dochain, D., Mouret, J., Vande Wouwer, A., and Sablayrolles, J.M. (2010). Dynamical modeling of alcoholic fermentation and its link with nitrogen consumption. In Proceedings of the 11th international symposium on computer applications in biotechnology (CAB 2010). Leuven, Belgium, 496-501.

de Gooijer, C., Bakker, W., Beeftink, H., and Tramper, J. (1996). Bioreactors in series: An overview of design procedures and practical applications. Enzyme and Microbial Technology, 18, 202-219.

Dochain, D. and Vanrolleghem, P. (2001). Dynamical Modelling and Estimation in Wastewater treatment Processes. IWA Publishing, U.K.

Doyle III, F.J. (1999). An anti-windup input-output linearization scheme for SISO systems. Journal of process control, 9, 213-220.

Gauthier, J. and Kupka, I. (2001). Deterministic Observation Theory and Applications. Cambridge University Press.

Harmand, J., Rapaport, A., and Trofino, A. (1999). Optimal design of two interconnected bioreactors-some new results. American Institute of Chemical Engineering Journal, 49, 1433-1450.

Hill, G. and Robinson, C. (1989). Minimum tank volumes for CFST bioreactors in series. The Canadian Journal of Chemical Engineering, 67, 818-824.

Katebi, R., Johnson, M., and Wilkie, J. (1999). Control and instrumentation for wastewater treatment plants. Springer, London.

Kendi, T.A. and Doyle III, F.J. (1997). An anti-windup scheme for multivariable nonlinear systems. Journal of process control, 7(5), 329-343.

Levenspiel, O. (1999). Chemical Reaction Engineering. John Wiley \& Sons, 3rd edition, New York.

Olsson, G. and Newell, B. (1999). Wastewater treatment systems: modelling, diagnosis and control. IWA publishing.

Rapaport, A. and Maloum, A. (2004). Design of exponential observers for nonlinear systems by embedding. International Journal of Robust and Nonlinear Control, 14, 273-288.

Simeonov, I., Stoyanov, S., et al. (2011). Modelling and extremum seeking control of a cascade of two anaerobic bioreactors. Int. J. Bioautomation, 15(1), 13-24.

Vigie, P., Goma, G., Renaud, P., Chamilothoris, G., Dahhou, B., and Pourciel, J. (1990). Adaptive predictive control of a multistage fermentation process. Biotechnology and bioengineering, 35(3), 217-223. 